\title{
Loggi: Treinamento Localizado em Automatização de Teste de Software em Ambiente Empresarial
}

\author{
Auri M. R. Vincenzi ${ }^{1}$, Ana P. S. Barbosa ${ }^{2}$, Cássio dos Santos Sousa ${ }^{2}$, \\ Cauê Polimanti ${ }^{2}$, Felipe Oliveira ${ }^{2}$, Gustavo de Paula ${ }^{2}$, José C. Maldonado ${ }^{2,3}$ \\ ${ }^{1}$ Departamento de Computação - Universidade Federal de São Carlos (UFSCar) \\ Caixa Postal 676 - 13565-905 - São Carlos - SP - Brasil \\ ${ }^{2}$ Loggi Tecnologia \\ São Paulo - SP - Brasil \\ ${ }^{3}$ Instituto de Ciências Matemáticas e de Computação - Universidade de São Paulo (USP) \\ Avenida Trabalhador São-carlense, 400 - 13566-590 - São Carlos - SP - Brasil

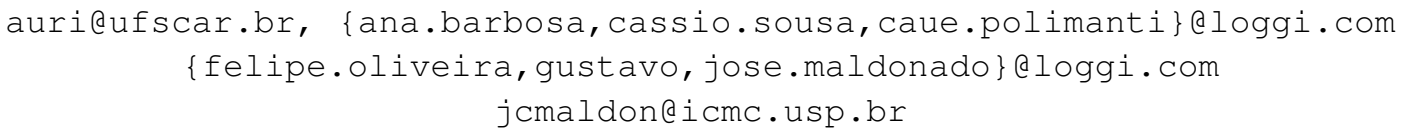

Resumo. A Loggi, criada em 2013, é uma das empresas unicórnios no Brasil, com soluções inovadoras e relevantes no setor de logística, com forte base em tecnologias digitais. O conhecimento teórico, o conhecimento de aspectos de automatização do processo e de evidências do custo e eficácia de critérios, técnicas e ferramentas de testes são de fundamental importância para a construção de produtos de software de qualidade. Salienta-se que dispor de material de treinamento e capacitação em automatização de teste de software, considerando essa vasta gama de tecnologias e ferramentas, é desafiador e indispensável. Neste artigo relata-se um treinamento bem sucedido em automatização em teste de software instanciado para o contexto da Loggi Tecnologia. O grande diferencial está na combinação de teoria com prática intensiva com base em produtos de software da empresa e ferramentas já adotadas no desenvolvimento das soluções na empresa.

\section{Introdução}

A Loggi, criada em 2013, é uma das empresas unicórnios no Brasil, com soluções inovadoras e relevantes no setor de logística, com forte base em tecnologias digitais. A Loggi tem se posicionado, de maneira inédita, para revolucionar o setor logístico no Brasil facilitando o crescimento de um novo comércio, com economia, agilidade e confiabilidade. A Loggi já atinge um número significativo de cidades do Brasil e já foram realizadas mais de 45 milhões de entregas. Até 2021, o objetivo é conectar todos os brasileiros, cobrindo 100 dos municípios, e almeja-se atingir cinco milhões de entregas por dia até o fim de 2025. A Loggi entende que a colaboração com a academia é frutífera na perspectiva de formação de recursos humanos, na atualização profissional e no avanço do conhecimento científico e tecnológico. Umas das perspectivas de colaboração é no treinamento in company em parceria com a academia. Com a expansão prevista, inclusive com a contratação de desenvolvedores de software, a Loggi tem por requisito aprimorar constantemente a qualidade dos processos e das soluções e aprimoramento constante de sua equipe. 
As atividades de Verificação, Validação e Teste (VV\&T) são fundamentais para o desenvolvimento de produtos de software de alta qualidade. Em particular, as atividades de Teste de Software representam um custo significativo para as empresas para o desenvolvimento e evolução de produtos de software ou de sistemas intensivos em software. Os custos variam em função do domínio de aplicação, podendo atingir valores superiores a $60 \%$ do custo total. O conhecimento do estado da arte e do estado da prática é fundamental para o estabelecimento de um processo e estratégias de teste eficazes e de baixo custo. Nessa perspectiva é fundamental o conhecimento teórico, o conhecimento de aspectos de automatização do processo e de evidências do custo e eficácia de critérios, técnicas e ferramentas, evidências essas obtidas a partir de estudos primários e secundários, com diretrizes de Engenharia de Software Experimental.

Na realidade, deve-se falar em Processo de Teste de Software, envolvendo atividades de planejamento, execução, documentação, análise de eficácia entre outras. Similarmente a modelos de maturidade de desenvolvimento de software, modelos de maturidade de processo de teste, tais como o Modelo TMMi [van Veenendaal and Wells 2012], desenvolvido pela TMMi Foundation, oferecem um guia e um framework para apoiar atividades de teste e melhoria de processo de teste de software.

Existe uma vasta gama de ferramentas proprietárias e de código aberto para apoio a diferentes atividades e critérios de teste. Desse modo, conciliar teoria e prática e evidências da eficácia das técnicas, critérios e ferramentas são pontos de fundamental importância para o planejamento e condução da atividade de teste com qualidade. Salienta-se que dispor de material de treinamento e capacitação em automatização de teste de software, considerando essa vasta gama de tecnologias e ferramentas, é desafiador e indispensável. Neste artigo relata-se um treinamento em automatização em teste de software instanciado para o contexto da Loggi Tecnologia.

\section{Treinamento em Teste de Software na Loggi Tecnologia}

Os grupos de teste de software do DC/UFSCar e ICMC/USP trabalham em parceria com outros grupos de pesquisa e pesquisadores no desenvolvimento de técnicas, critérios, processos e estratégias de teste, ferramentas de apoio, e material de treinamento e capacitação. Com base nesse conhecimento e evolução, foram desenvolvidos mais de 300 horas de material livre de treinamento e capacitação com base em ferramentas open source [J. R. H. Carvalho et al. 2018]. Em decorrência desse treinamento, publicou-se o livro "Automatização de Teste de Software com Ferramentas de Software Livre" [Vincenzi et al. 2018]. Em um livro prévio desses grupos, "Introdução ao Teste de Software" [Delamaro et al. 2016], os autores apresentam conceitos teóricos sobre teste de software e exemplos didáticos.

No contexto da empresa Loggi, o foco do treinamento era o de capacitar os desenvolvedores da Loggi (Loggers) com aspectos teóricos e práticos em teste de software, mas utilizando as ferramentas adotadas no escopo da empresa, visando ao aprimoramento dos processos e de estratégias de teste. ) material existente foi, em uma primeira etapa, revisitado, juntamente com os Loggers, e foram definidos os tópicos de interesse e as ferramentas que seriam utilizadas no curso, assim como alguns produtos de software da própria Loggi como objeto de teste. Ênfase foi dada a conceitos presentes no TMMi [van Veenendaal and Wells 2012], aderentes às necessidades da empresa. 
Após a identificação dos tópicos e das ferramentas, o curso foi dividido em um conjunto de aulas teóricas e práticas, com as ferramentas selecionadas. O material para as aulas teóricas foi readequado. O material para as aulas práticas foram elaborados com base nas ferramentas e produtos Loggi. As aulas práticas seriam ministradas pelos Loggers, com planejamento prévio conjunto.

A Figura 1 apresenta os tópicos e a distribuição entre teoria e prática. Cada um dos módulos teóricos tinha a duração de 3 horas e 30 minutos com exemplos de uso de ferramentas, considerando a tecnologia Java. Os módulos práticos, também com a mesma duração, utilizavam ferramentas com funcionalidades equivalentes mas para a tecnologia adotada pela empresa (Python ou Javascript).

\begin{tabular}{|c|c|}
\hline 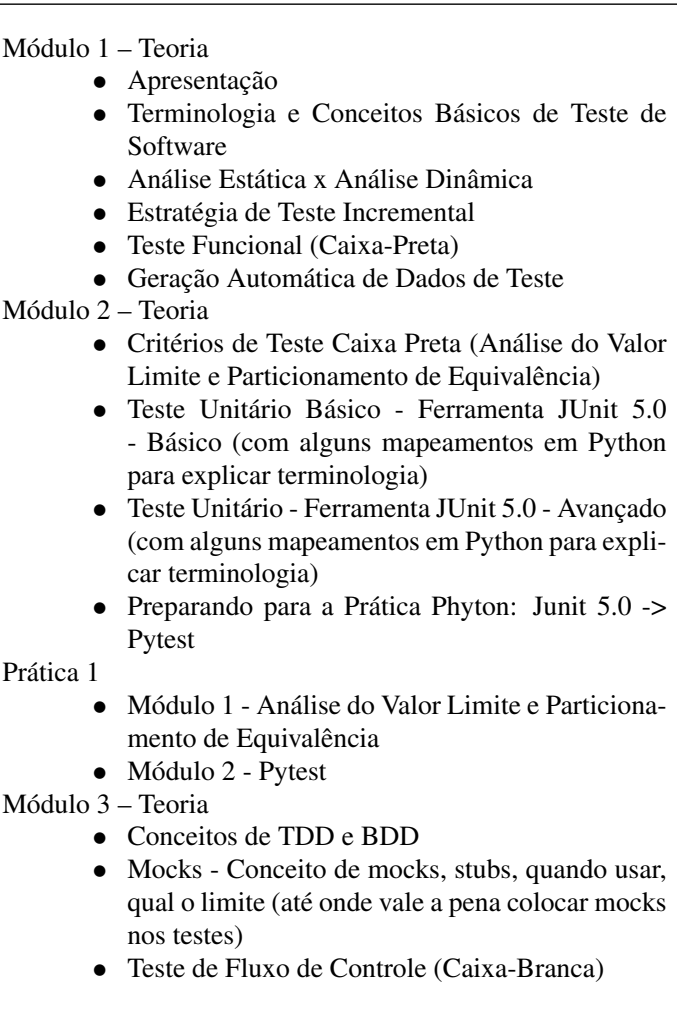 & 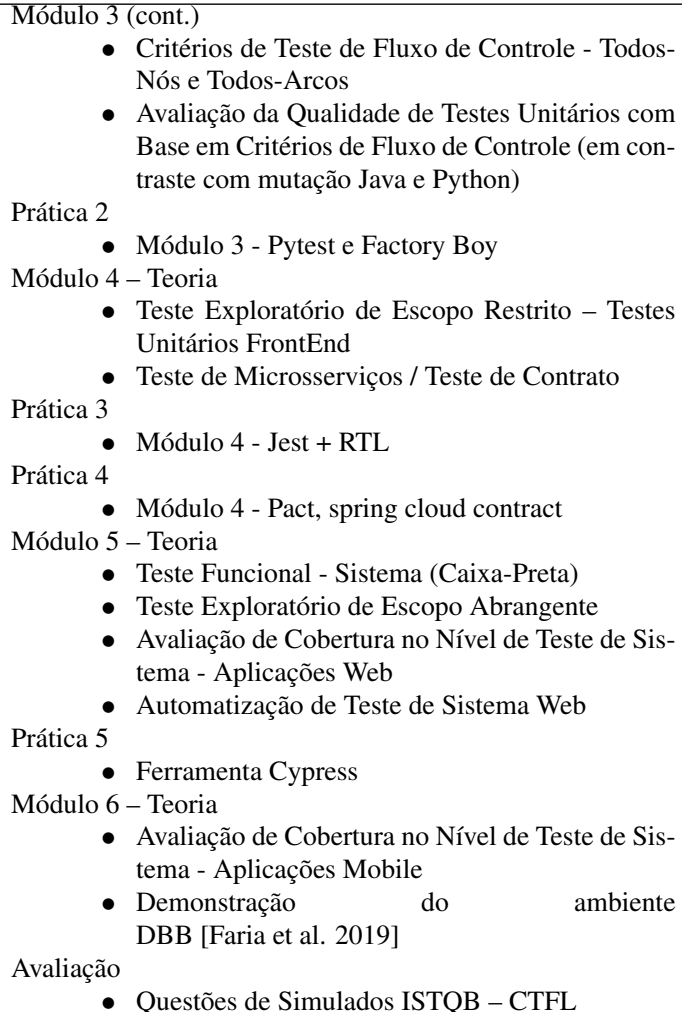 \\
\hline
\end{tabular}

Figura 1. Conteúdo Programático Intercalando Teoria e Prática

Inicialmente as aulas, tanto teórica como as práticas seriam oferecidas presencialmente, com hands on nas aulas práticas. No entanto, após a Prática 1 do curso, em função da pandemia, a Loggi determinou que as atividades fossem realizadas em home office. Mesmo com esse contexto, manteve-se a oferta do curso de forma virtual. Essencialmente, não houve prejuízo na execução do curso, pois todos tinham acesso remoto ao ambiente de desenvolvimento da Loggi e puderam executar as atividades práticas remotamente tanto de forma síncrona como assíncrona.

Um diferencial importante do treinamento foi a possibilidade dos Loggers utilizarem nas práticas as ferramentas de teste adotadas pela empresa, além de empregarem os conceitos teóricos e práticos nos sistemas reais disponibilizados e utilizados pela Loggi.

Optou-se pela realização de avaliações com base em questões de simulados para certificação em teste de software - CTFL do ISTQB, o que propiciou aos participantes 
não apenas o entendimento da evolução que tiverem em termos de conceitos e ferramentas de teste, mas também uma noção da exigência para a certificação, motivando a maioria a tentar a certificação CTFL futuramente. Trinta e quatro loggers foram aprovados e obtiveram o certificado da UFSCar. No final, os participantes conduziram uma avaliação da qualidade e efetividade do curso oferecido.

\section{Considerações Finais}

Neste artigo apresentou-se um relato sucinto de um treinamento em automatização de teste de software bem sucedido, realizado por meio de uma parceria da academia com a empresa Loggi. O grande diferencial foi a combinação de teoria com prática intensiva com base em produtos de software da empresa e ferramentas já adotadas no desenvolvimento das soluções na empresa. Todos os participantes puderam nivelar, aprofundar e homogeneizar seus conhecimentos em teste de software e ferramentas de apoio, voltados para o seu dia a dia. Os participantes podem se tornar multiplicadores do conhecimento no âmbito da Loggi e avaliaram positivamente a iniciativa.

Espera-se que na mesma linha do material de treinamento e capacitação desenvolvido com o foco em ferramentas para Java tenha-se também material adicional disponibilizado para a comunidade, considerando a linguagem Python e Javascript. Ainda, esse tipo de material motiva o ensino no ambiente acadêmico com o uso de produtos de software da indústria.

Um desdobramento dessa parceria será o envolvimento da Loggi em estudos experimentais em um projeto de pesquisa e desenvolvimento sobre automatização de teste de software, avaliando custo e eficácia de estratégias e ambientes de teste em contextos industriais. Finalmente, com os conhecimentos adquiridos, os Loggers têm capacidade de evoluir o processo de teste adotado pela empresa sempre que necessário, com base em métricas, avaliando a relação custo benefício das possíveis alterações realizadas.

\section{Referências}

Delamaro, M. E., Maldonado, J. C., and Jino, M. (2016). Introdução ao Teste de Software. Elsevier, Rio de Janeiro, RJ, 2 edition.

Faria, K. A. C., de Aquino Gomes, R., de Andrade Freitas, E. N., and Vincenzi, A. M. R. (2019). On using collaborative economy for test cost reduction in high fragmented environments. Future Generation Computer Systems, 95:502-510.

J. R. H. Carvalho, A. Vnicenzi, J. C. Maldonado, and M. Gonçalves (2018). Industry and Academia Partnership for Short-time High-level Qualification. In 2018 IEEE Frontiers in Education Conference (FIE), pages 1-8. Journal Abbreviation: 2018 IEEE Frontiers in Education Conference (FIE).

van Veenendaal, E. and Wells, B. (2012). Test Maturity Model integration TMMi (Guidelines for Test Process Improvement). UTN Publishers, Den Bosch, The Netherlands.

Vincenzi, A. M. R., Delamaro, M. E., Dias Neto, A. C., Fabbri, S. C. P. F., Jino, M., and Maldonado, J. C. (2018). Automatização de Teste de Software com Ferramentas de Software Livre. SBC. Elsevier. bibtex*[chapter=Ferramentas de Geração Automática de Dados de Teste]. 